\title{
A randomised controlled trial of a patient based Diabetes recall and Management system: the DREAM trial: A study protocol [ISRCTN32042030]
}

\author{
Martin Eccles ${ }^{1}$, Gillian Hawthorne ${ }^{2}$, Paula Whitty*3, Nick Steen ${ }^{1}$, \\ Alessandra Vanoli ${ }^{1}$, Jeremy Grimshaw ${ }^{4}$ and Linda Wood 5
}

Address: ${ }^{1}$ Centre for Health Services Research, University of Newcastle, Newcastle upon Tyne, UK, ${ }^{2}$ Diabetes Centre, Newcastle Primary Care Trust, Newcastle upon Tyne, UK, ${ }^{3}$ Department of Epidemiology and Public Health, University of Newcastle, Newcastle upon Tyne, UK, ${ }^{4}$ Ottawa Health Research Institute, University of Ottawa, Ottawa, Canada and ${ }^{5}$ Northern and Yorkshire Regional Office, Diabetes UK, Darlington, UK

E-mail: Martin Eccles - martin.eccles@ncl.ac.uk; Gillian Hawthorne - Gillian.Hawthorne@ncht.northy.nhs.uk; Paula Whitty* - p.m.whitty@ncl.ac.uk; Nick Steen - I.N.Steen@ncl.ac.uk; Alessandra Vanoli - Alessandra.Vanoli@ncl.ac.uk; Jeremy Grimshaw - igrimshaw@ohri.ca; Linda Wood - northandyorks@diabetes.org.uk

*Corresponding author

Published: 2 I March 2002

BMC Health Services Research 2002, 2:5

This article is available from: http://www.biomedcentral.com// 472-6963/2/5

(C) 2002 Eccles et al; licensee BioMed Central Ltd. Verbatim copying and redistribution of this article are permitted in any medium for any purpose, provided this notice is preserved along with the article's original URL.
Received: |4 December 200|

Accepted: 21 March 2002

\begin{abstract}
Background: Whilst there is broad agreement on what constitutes high quality health care for people with diabetes, there is little consensus on the most efficient way of delivering it. Structured recall systems can improve the quality of care but the systems evaluated to date have been of limited sophistication and the evaluations have been carried out in small numbers of relatively unrepresentative settings. Hartlepool, Easington and Stockton currently operate a computerised diabetes register which has to date produced improvements in the quality of care but performance has now plateaued leaving substantial scope for further improvement. This study will evaluate the effectiveness and efficiency of an area wide 'extended' system incorporating a full structured recall and management system, actively involving patients and including clinical management prompts to primary care clinicians based on locally-adapted evidence based guidelines.
\end{abstract}

Methods: The study design is a two-armed cluster randomised controlled trial of 61 practices incorporating evaluations of the effectiveness of the system, its economic impact and its impact on patient wellbeing and functioning.

\section{Background}

\section{Delivering care to people with diabetes}

There is broad, international agreement over what constitutes high quality health care for people with diabetes $[1,2]$. This will be enshrined in a National Service Framework for people with diabetes, due in summer 2002. However, in the face of poor current performance $[3,4]$ the most efficient method of delivering care remains un- clear [4]. Following a 1994 systematic literature review [5] suggesting structured care improved patient care, an editorial in the British Medical Journal concluded that more evaluative research was needed before widespread adoption of any of the models could be recommended [6]. A subsequent systematic review of routine surveillance of patients with diabetes by Griffin and Kinmonth [7] concluded "Computerised central recall, with prompting for pa- 
tients and their family doctors, can achieve standards of care as good or better than hospital outpatient care, at least in the short term. The evidence supports provision of regular prompted recall and review of people with diabetes by willing general practitioners and demonstrates that this can be achieved, if suitable organisation is in place'. However, the evidence base on which these conclusions are based is limited in several ways. Firstly there are only five randomised controlled trials (RCTs) involving 1058 patients. All of these studies are 'patient randomised" trials, thus potentially under-estimating the effectiveness of the intervention (see Study Design). They were all evaluating more or less selected patients and general practices and none of them were explicitly evaluating a UK National Health Service (NHS) service area wide intervention. Only one of the four UK based studies evaluated patient based outcomes and included an economic assessment and this study only involved patients from three general practices [8]. Thus, the effectiveness of an area wide, patient focussed, structured recall and management system (in terms of process of care, patient outcome and economic impact) remains unknown.

\section{The current system}

The current computerised diabetes management system runs in Hartlepool, Easington and Stockton, three Primary Care Group (PCG) areas, in the Northern and Yorkshire Region. It was introduced to all 36 general practices in Hartlepool and Easington Districts in mid-1995. Stockton (25 practices) agreed to join the system in 1999 and it was operational there by October 2000 . There are three key components to the current system:

\section{A central register of patients with diabetes.}

2. A structured minimum dataset to be completed and returned to the central register.

3. The provision of both patient specific and aggregated data to both patients and clinicians.

The system (developed by Westman Medical Software) allows three methods of collection of data at each contact with a patient with diabetes who is registered on the database. Two methods use a standard form completed by clinicians to collect data concordant with the UK minimum data set [9]. Within secondary care, forms are completed at every new patient or annual review. In primary care, forms are completed by the practice nurse (usually) or general practitioner, either opportunistically or at practice diabetic clinics. In both cases, the completed data forms are sent to the Diabetes Register Facilitator for data entry. Thirdly, the hospital laboratory provides a monthly download of laboratory test details (e.g. HbA1c). A patient can be identified as having diabetes and added to the register by any permutation of one or more of these three routes. Feedback of individual patients' data, including review status, is provided to general practices quarterly. This feedback is 'passive' in that it does not explicitly prompt either patients or doctors as to required actions. Audit packages within the software can audit on every variable collected. District wide audit is provided on anonymised aggregated data; individual practice audits (with comparisons to other practices) are provided to participating practices at least annually. Feedback of the data to the patient (for hospital patients only) is by a patient information sheet and to the GP as a standardised letter. A Diabetes Register Facilitator co-ordinates and updates the register. A steering group composed of $\mathrm{GH}$, the Diabetes Register Facilitator and representatives of the PCGs and patients, oversees the register and deals with issues such as confidentiality.

\section{Impact of the system to date}

Measures of the impact of the system to date relate only to Hartlepool, Easington and Stockton. The main impact on patient registration was in its first 12 to 18 months of operation: during 1995, 747 patients were registered on the system ( $0.4 \%$ prevalence) which had increased to 3867 (1.8\% prevalence) by the end of 1996 . The increase in registration has stabilised since then, reaching 4324 (2\% prevalence) by 1999 . During $1999,70 \%$ of registered patients attended a clinic; $52 \%$ had their feet examined and $51 \%$ had their eyes examined. Seventy three per cent had an HbA1c result recorded and $69 \%$ a blood pressure measurement. These figures are similar to those reported by other centres using the same system [10].

\section{The need for an extended system}

Recording of clinical measures increased during the first few years of operation of the system but began to plateau more recently (for example, $50 \%$ of patients had an HbA1c recorded during 1996, compared to 60\% in 1997 and $63 \%$ in 1998). This plateauing of performance has been reported by others [10]. We believe that this is due to a lack of coordination (patients being lost to follow up) and lack of prompting of clinicians to deliver appropriate clinical interventions. Furthermore, given that most patients with diabetes are primarily seen in primary care the greatest potential impact is from optimising and extending the system in primary care. In order to address these shortcomings the additional key components, over and above those already in the system, will be:

1. Locally adapted evidence based guidelines for the management and follow up of patients with diabetes.

2. Automated prompts to patients and primary care clinicians that a review consultation is necessary. 
3. A structured management sheet (including patient specific management suggestions based on (1)).

4. An enhanced monitoring system to follow up reasons for non-attendance from both patients and clinicians and to re-schedule appointments, based on nonreturn of a completed management sheet.

\section{Patient feedback for patients in primary care.}

There is some limited supportive trial evidence for these developments, although the existing studies involved small sample sizes and may not be generalisable to the NHS [11-13]. In evaluating the system with these extended features this study will also address the design shortcomings of previous studies of shared care in diabetes [6]. It will be tailored to each practice, PCG defined areas will be studied, rather than an unrepresentative sample of general practices; and the system will be transparent and replicable in other areas.

\section{Methods}

\section{Design of the study}

The study design is a pragmatic two-arm cluster randomised controlled trial. The unit of randomisation will be the general practice. Simple patient randomised trials are rightly considered the most robust method of assessing most health care innovations [14]. This design, however, cannot be regarded as the gold standard for evaluating systematic approaches to chronic disease management, an essentially behavioural field of research $[15,16]$. If both intervention and control patients were to be cared for within the same practice there is the risk that the management of control patients would be influenced by the practitioners knowledge of the care of intervention patients. This would result in an underestimation of the effect of the intervention [17]. Therefore, practices rather than patients are the appropriate unit of randomisation and analysis.

As the current system has been in place for different lengths of time within the three participating PCGs, we will stratify the randomisation by PCG. Randomisation will be performed by a statistician independent of the research team using computer generated numbers to avoid allocation bias [18].

\section{Study setting and recruitment of practices}

The study will be based in the general practices of the three PCGs of Easington, Hartlepool and Stockton. Since the recent merger of Hartlepool and North Tees Acute Trusts all three PCGs are now exclusively served by one secondary care diabetes service (and thus the one diabetes register). GH is the lead clinician for diabetes services in the new Trust.
The 61 general practices in the three PCGs constitute the target practices for the study and we will attempt to recruit all practices. The PCG diabetes leads or the PCG clinical governance leads in all three PCGs have provided letters confirming their support for the project. We do not envisage major difficulties with recruitment, given the need to agree local guidelines as part of the process involved in the Trust merger, the likely requirements in the forthcoming National Service Framework for diabetes, and the 100\% practice coverage with the current diabetes system. We will (through the PCGs) write to all practices, giving information about the project to the senior partner or diabetes lead and practice manager of practices. Practices will be invited to opt out if they do not wish to be included in the study - this is an approach we have used successfully before. The PCG diabetes lead, clinical governance lead and $\mathrm{GH}$ will be co-signatories of this letter. If practices do decline we will collect data on characteristics of non-participating practices to assess the impact on the generalisability of the trial's findings. Finally, if there are significant problems with recruitment, there are other practices which could be approached in a nearby PCG (South Tyneside) which uses the same software for its diabetes register.

\section{Details of the intervention}

Local guidelines and management prompts

A guideline development group will be established to develop local guidelines for the management of diabetes, based upon available evidence based guidelines (Scottish Intercollegiate Guidelines Network [19-22](SIGN, 1996, 1997a, 1997b, 1997c), and Effective Care Bulletins $[23,24]$. They will also use the forthcoming national diabetes guidelines as these become available. The group will be multidisciplinary and contain primary and secondary care doctors and nurses, patients and the Diabetes Register Facilitator [25]. The group will define review periods for specified patient groups (e.g. patients with diabetes satisfactorily controlled on diet alone should be reviewed every 12 months), referral criteria for patients moving from primary to secondary care and back and simple decision rules for the management prompts. These would be of two types. The first would prompt for actions to be performed and only require their performance to be documented (e.g. asking for a foot examination to be performed in a patient who does not have a recorded foot examination). The second would be more complex and suggest alterations to clinical management on the basis of data in the database (e.g. patients with persistently raised blood pressure should have their anti-hypertensive medication increased). These decision rules will be integrated into the recall and management system. 


\section{Running the system}

The proposed enhancements to the system are designed to require the primary care team to perform no additional work over and above the current configuration. The current database has a patient identifier, a minimum dataset and retrieval systems to support the structured recall of patients. Westman Medical Software has agreed to amend the system as required. A 'circle of information exchange' will be established between the participating general practices and the database. The local guidelines will be used to adapt the current centralised database, along with the practices' preferred method of following up patients (for example, within consultations in routine surgeries or within special clinics). The central database system will identify when patients are due for review (based upon the local guidelines) and will generate a letter to the patient asking them to make an appointment for a review consultation. Patient information or educational materials could be included with the letter. At the same time, the central database will generate a letter to the practice stating that the patient should be making a review appointment in the near future. The letter to the practice will include a management sheet (to be held in the patient's record) to capture an agreed minimum data set to be collected during the consultation. This management sheet will also contain the relevant prompts (as described above).

When the patient is seen in the practice, the primary care professional (currently this is usually done by the practice nurse) will complete the management sheet and return a copy for entry onto the central register within a designated period of time. This circle of information is broken if the patient does not visit the general practice as planned or the general practice does not return the management sheet to the central register. If this happens, the central register would alert the Diabetes Register Facilitator who will ascertain the reason for failure and take appropriate action, (e.g. send a reminder to the patient, prompt the practice to return the management sheet).

A range of educational activities will be provided for intervention practices, as part of the usual local structures for contact with practices, with some additions, These will include: distribution of information about the trial in local newsletters; meetings with practice clinical governance leads; evening meetings for practice nurses (with small group discussion of the practical implications for intervention practices); and a telephone meeting with the practice diabetes lead (usually the practice nurse) in each intervention practice.

Practices in the control arm will continue to receive the recall system as currently configured.

\section{Logistical considerations}

From the prevalence of patients with diabetes on the current register, there will be about 7500 patients on the system if 61 practices are recruited. Half of these will be in intervention practices. On current patterns of usage, we anticipate there being the need for 1.5 recalls per annum per patient on the register, resulting in about 6000 recalls per year for the intervention group. Assuming a 40 week working year, the system will need to dispatch, receive and process about 150 forms per group per week.

\section{Identification of patients}

Patients for the structured recall and management system are already identified on the Hartlepool and North Tees database. As some practices have children registered on the system, who are under the care of an exclusively secondary care adolescent service, an age limit of 18 years or over will be set for inclusion. Practices will be asked to check lists of their patients on the database regularly throughout the study. The central database will remove patients from the recall system who are known to have died or moved away.

\section{Patient consent}

Patients have already consented, or are being consented, to their data being held within the current diabetes register. The study will involve no extra 'routine' data being collected, and this data will be anonymised before being sent for analysis; all data held for analysis will be held in accordance with the Data Protection Act. For the patientbased questionnaire study, we will seek additional patient consent to complete one survey. The three relevant Local Research Ethics Committees have approved the trial.

\section{Data collection}

The main study outcome measures will be rates of performance of process of care and the patient based measures of functional and psychosocial wellbeing. Data will be collected for 15 months after the start of the intervention. Fifteen months was chosen to allow for patients who are reviewed every 12 months but fail to attend on initial invitation.

\section{Process of care variables}

Process of care variables will be collected via the computerised database. The exact data to be collected will be determined by both the current content of the database and the guidelines but will include such data items as rates of attendance at clinics and annual reviews, conduct of eye and feet examinations, performance of investigations and prescribing. We will also collect data on clinical measures (e.g. HbA1c, and blood pressure levels). 


\section{Outcome of care measures}

Outcome of care data will be collected, by postal questionnaire, 15 months after commencement of the study. A portfolio of validated [26-30] and responsive [30,31] generic and disease specific instruments will be used to measure functional and psychosocial variables that will be potentially influenced by the intervention. These will include:

i) The SF36 health status profile which we will use to generate Mental (MCS) and Physical Component Summary Scales (PCS) $[26,27,32]$.

ii) The Newcastle Diabetes Symptoms Questionnaire [28].

iii) The Bradley Treatment Satisfaction Questionnaire [29].

Patient costs questions will be developed by the study health economist. We have successfully used such packages of questionnaires within trials before and have achieved response rates in excess of $70 \%$ in similar surveys in this region. [33].

\section{Sample size considerations}

On the basis of previous work we have made the following assumptions. The mean number of patients per practice for whom we will be able to collect process data will be 30 and the ICC (a measure of the lack of independence of responses from patients from the same practice) calculated from our local data is 0.14 for measures of process (whether a blood pressure measurement and whether an HbA1c measurement has been recorded in a 12 month period). Standard methods for determining the sample size requirements for a cluster randomised trial [34] indicate that we need 60 practices to detect a difference of $15 \%$ $(42.5 \% \mathrm{v} 57.5 \%)$ with $80 \%$ power assuming a significance level of $5 \%$. Assessment of outcome of care will be based on health status scales such as the SF-36. Previous work has shown that this type of intervention is likely to produce an effect size of approximately 0.25 in such measures [32] and that the ICCs for such measures will be approximately 0.07 . The most efficient study design (that minimises the number of patients required) is one that makes use of all the available practices. A sample of 27 patients from each of 61 practices will give us $85 \%$ power to detect an effect size of 0.25 assuming a significance level of $5 \%$. With a predicted response rate of approximately $70 \%$ (based on our experience in the COGENT study [33]) after two reminders, our starting sample size will need to be 2379 patients (approximately 39 patients per practice).

\section{Principles of data analysis}

Analysis will be by intention to treat. Multilevel modelling (using the MlwiN package [35]) will be used to take into account the clustering of patients within practices [36]. Both binary variables (when a process was undertaken or not) and continuous variables (such as the physical health component of the SF-36) can be analysed using these techniques. For both types of variable, variation between practices will be fitted as a random effect and the difference between intervention and control practices will be fitted as a fixed effect. In the case of binary variables, a logit link function will be used.

\section{Economic evaluation}

The economic impact of implementing the new structured recall and management system will be evaluated in terms of the marginal costs of adapting and running the system; the costs of developing and disseminating the guidelines; the educational activities for intervention practices; the implications for the use of health care services; and the costs to the patients and their carers. The benefits will be measured as described earlier on in the clinical study. The estimation of health service resource use will relate to diabetes-specific clinical visits, tests, investigations, and procedures. This data will be routinely collected as part of the management system implementation and subsequent costing, using health service pay and price data, will be undertaken using a mixed approach based on micro-costing and gross-costing methods [37]. Use of drugs, referrals to secondary care and the impact of the intervention on the change of use of patients' and their carers' time will also be monitored through postal questionnaires at the end of the follow-up period. A sensitivity analysis will be undertaken to test the robustness of the results to the uncertainty not related to sampling variations and to enhance the generalisability of the results [38]. We are aware that the costs of the system might be balanced only in the longer term against the cost savings related to averted complications [39-41]. However, the assessment of the benefits in terms of final outcomes (e.g lives saved, or QALYs) and long term costs is beyond the objective of the present study.

\section{Competing interests}

None declared

\section{Acknowledgements}

The study is funded by Diabetes UK and the Northern and Yorkshire NHS Executive Research and Development Programme. We thank our collaborators at North Tees and Hartlepool NHS Trust, particularly Dr Carr, Dr MacLeod, Joanne Clayton and John Fitzsimmons; at Easington Primary Care Group and North Tees Primary Care Trust; and at South Tyneside Primary Care Group and South Tyneside Healthcare NHS Trust, particularly Drs Bradshaw and Parr, Clare Beard and Wynn Schembrie. 


\section{References}

I. The Acropolis Affirmation: Diabetes Care - St Vincent in Progress. Statement from St Vincent Declaration meeting, Athens, Greece, March 1995. Diabetic Medicine 1995, I 2:636

2. UK Prospective Diabetes Study (UKPDS) Group: Intensive blood glucose control with sulphonylureas or insulin compared to conventional treatment and risk of complications in patients with type 2 diabetes (UKPDS 33). Lancet 1998, 352:837-853

3. Audit Commission: Testing Times: A Review of Diabetes Services in England and Wales. London: Audit Commission, 2000

4. Diabetes UK Report: Diabetes in the UK - the missing million. Perceptions and reality of diabetes today. London: Diabetes UK, 2000

5. Greenhalgh PM: Shared care for diabetes: a systematic review. Royal College of General Practitioners 1994 (occasional paper 67). London: Royal College of General Practitioners, 1994

6. Sowden AJ, Sheldon TA, Alberti G: Shared care in diabetes (editorial). $B M]$ 1995, 3 I 0: | 42-143

7. Griffin S, Kinmonth AL: Diabetes care: the effectiveness of systems for routine surveillance for people with diabetes (Cochrane Review). In: The Cochrane Library, Issue 4, 1999. Oxford: Update Software, 1999

8. Diabetes Integrated Care Evaluation Team (DICE): Intergrated care of diabetes: clinical, psychosocial and economic evaluation. BMJ 1994, 308: I 208-1212

9. Vaughan NJ, Home PD: The UK Diabetes Dataset: a standard for information exchange. Diabetes Audit Working Group of the Research Unit of the Royal College of Physicians. British Diabetic Association. Diabetic Medicine 1995, I 2:71 7-722

10. New JP, Hollis S, Campbell F, McDowell D, Burns E, Dornan TL, Young RJ: Measuring clinical performance and outcomes from diabetes information systems: an observational study. Diabetologia 2000, 43:836-843

II. Lobach DF, Hammond LS: Computerised decision support based on a clinical practice guideline improves compliance with care standards. Am J Med I997, I 02:89-98

12. Nilasena DS, Lincoln MJ: A computer-generated reminder system improves physician compliance with diabetes preventive care guidelines. Proceedings - the Annual Symposium on Computer Applications in Medical Care 1995, 640-645

13. Peters AL, Davidson MB: Application of a Diabetes Managed Care Program: the feasibility of using nurses and a computer system to provide effective care. Diabetes Care 1998, 21:10371043

14. Cochrane A: Effectiveness and efficiency: random reflections on health services. London: Nuffield Provincial Hospitals Trust, 1972

15. Cook TD, Campbell DT: Quasi-experimentation: design and analysis issues for field settings. Chicago: Rand McNally, 1979

16. Grimshaw JM, Campbell MK, Eccles MP, Steen IN: Experimental and quasi-experimental designs for evaluating guideline implementation strategies. Family Practice 2000, I7:SI I-SI 8

17. Morgan M, Studney DR, Barnett GO, Winickoff RN: Computerised concurrent review of prenatal care. Quality Review Bulletin 1978, 4:33-36

18. Schultz KF, Chalmers I, Hayes RJ, Altman DG: Empirical evidence of bias: dimensions of methodological quality associated with estimated of treatment effects in controlled trials. JAMA 1995, 273:408-4I2

19. Scottish Intercollegiate Guidelines Network (SIGN): Prevention of visual impairment in diabetes and minimum dataset collection in diabetes patients. Guideline no 4. 1996

20. Scottish Intercollegiate Guidelines Network (SIGN): Management of diabetic renal disease. Guideline no I I. 1997

21. Scottish Intercollegiate Guidelines Network (SIGN): Management of diabetic foot disease. Guideline no 12. 1997

22. Scottish Intercollegiate Guidelines Network (SIGN): Management of cardiovascular disease. Guideline no 19. 1997

23. NHS Centre for Reviews and Dissemination, University of York: Complications of Diabetes: Screening for Retinopathy. Management of Foot Ulcers. 1999, 5(4):

24. NHS Centre for Reviews and Dissemination, University of York: Complications of diabetes: Renal disease and promotion of self-management. 2000, 6(I):

25. Corner L, Curless R, Parker SG, Eccles M, Gregson B, Bond J, James OFW: Developing guidelines for day hospitals for older peo- ple: adaptation of national guidelines for local use. Journal of Clinical Effectiveness 1998, 3:10-13

26. Ware JE, Snow KK, Kosinski M, Gandek B: SF-36 Health Survey Manual and Interpretation Guide. The Health Institute, New England Medical Centre. Boston, Massachusetts: The Health Institute, New England Medical Centre, 1993

27. Ware JE, Kosinski M, Keller SD: SF-36 Physical and Mental Health Summary Scales: A User's Manual. The Health Institute, New England Medical Centre. Boston, Massachusetts: The Health Institute, New England Medical Centre, 1994

28. McColl E, Steen IN, Meadows KA, Hutchinson A, Eccles MP, Hewison J, Fowler P, Blades SM: Developing outcome measures for ambulatory care - an application to asthma and diabetes. Soc Sci Med 1995, 4 I ( I 0): I 339-1348

29. Bradley C, Ed: The Diabetes Treatment Satisfaction Questionnaire (DTSQ). In: Handbook of Psychology and Diabetes: A Guide to Psychological Measurement in Diabetes Research and Practice. 1994

30. Tilly K, Belton A: Continuous monitoring of health status outcomes: experience with a diabetes education program. Diabetes Educator 1995, 21:413-419

31. Whitty P, Steen IN, Eccles M, McColl E, Hewison J, Meadows K, Clapp $Z$, Hutchinson A: A new self-completion outcome measure for diabetes: is it responsive to change? Quality of Life Research 1997, 6:407-413

32. Jenkinson C, Layte R, Wright L, Coulter A: The UK SF-36: an analysis and interpretation manual. Oxford, Health Services Research Unit: University of Oxford, 1996

33. Eccles MP, Grimshaw JM, Steen N, Parkin D, Purves I, McColl E, Rousseau $N$ : The design and analysis of a randomised controlled trial to evaluate computerised decision support: the $\mathrm{CO}$ GENT Study. Family Practice 2000, I 7:180-186

34. Dormer A, Birkett N, Buck C: Randomisation by cluster: sample size requirements and analysis. Am J Epidem I98I, I | 4:906-9|4

35. Goldstein H, Rasbash J, Plewis I, Draper D, Browne W, Yang M, Woodhouse G, Healy M: A user's guide to MLwiN. Multilevel Models Project. London: University of London, 1998

36. Goldstein H: Multilevel Statistical Models London : Arnold, 1995

37. Raftery J: Costing in economic evaluation. BMJ 2000, 320: 1597

38. Briggs $A H$, Gray $A M$ : Handling uncertainty when performing economic evaluation of healthcare interventions. Health Technology Assessment 1999, 3(2):

39. Ginsberg BH, Tan MH, Mazze R, Bergelson A: Staged diabetes management: computerizing a disease state management program. Journal of Medical Systems 1998, 22:77-87

40. Brown JB, Nichols GA, Glauber HS, Bakst AW: Type 2 Diabetes: Incremental medical care costs during the first 8 years after diagnosis. Diabetes Care 1999, 22: I | |6-| | 24

4I. Brown JP, Pedula KL, Bakst AW: The progressive cost of complications in Type 2 diabetes mellitus. Archives of Internal Medicine 1999, 1 59:1873-1880

\section{Pre-publication history}

The pre-publication history for this paper can be accessed here:

http://www.biomedcentral.com/1472-6963/2/5/prepub 